\title{
Community outbreak of invasive group $A$ streptococcus infection in Ontario, Canada
}

\author{
C Dickson ${ }^{1}$, MT Pham ${ }^{2 *}$, V Nguyen ${ }^{2}$, C Brubacher ${ }^{2}$, MS Silverman ${ }^{3,4}$, K Khaled $^{2}$, G Hovhannisyan ${ }^{2}$
}

\section{Abstract}

Background: Outbreaks of invasive group A streptococcal infection (iGAS) have historically occurred in institutional settings. Increasingly, community-based outbreaks have been reported, often among marginalized populations, yet few guidelines exist for managing iGAS outbreaks in such settings.

Objective: To describe the ongoing outbreak of iGAS in Middlesex-London, Ontario, and the challenges that arose while applying current guidelines to a marginalized population in a community setting.

Methods: The outbreak investigation included all iGAS cases in Middlesex-London with an onset date from April 1, 2016 to February 28, 2018. Clinical specimens were submitted to provincial and federal laboratories for typing. Public health management of the outbreak involved environmental health inspections, contact tracing, chemoprophylaxis of close contacts, swabbing to determine colonization rates of Streptococcus pyogenes, and communicating with stakeholders and the public.

Results: A total of 156 confirmed cases of iGAS corresponding to 147 individuals were reported in less than two years. More than $60 \%$ of cases occurred in men $(n=91)$ and almost half $(n=71)$ of the total number of cases were persons who used drugs (PWUD) and/or were under-housed. Of the PWUD cases, 58 of 65 (89\%) used injection drugs. Key challenges in controlling this outbreak included reaching PWUD and under-housed people; completing a case history and contact list; facilitating completion of treatment; dealing with concurrent infections such as human immunodeficiency virus (HIV) and hepatitis $\mathrm{C}$ virus (HCV); and optimizing environmental health conditions. Guidelines were adapted so contacts who shared drugs or injection drug equipment with a known iGAS case would be offered chemoprophylaxis regardless of the clinical severity of the case. To optimize treatment completion, a single-dose of azithromycin for individuals in close contact with PWUD and/or under-housed cases was given. Cases with macrolide-resistant strain emm9 have recently emerged.

Conclusion: The application of institution-based guidelines for iGAS outbreaks has been ineffective in controlling this particular community outbreak. There is a need for guidelines on managing outbreaks of iGAS in the community especially when an outbreak involves marginalized populations.

\author{
Affiliations \\ ${ }^{1}$ Canadian Field Epidemiology \\ Program, Public Health Agency of \\ Canada, Ottawa, ON \\ ${ }^{2}$ Middlesex-London Health Unit, \\ London, ON \\ ${ }^{3}$ Division of Infectious Diseases, \\ Schulich School of Medicine \& \\ Dentistry, Western University, \\ London, ON
}

*Correspondence: mai.pham@ mlhu.on.ca

Suggested citation: Dickson C, Pham MT, Nguyen V, Broacher C, Silverman MS, Khaled K, Hovhannisyan G. Community outbreak of invasive group A streptococcus infection in Ontario, Canada. Can Commun Dis Rep 2018;44(7/8):182-8. https://doi.org/10.14745/ccdr.v44i78a06

Keywords: invasive group A streptococcus, outbreak, community, marginalized population, persons who use drugs

\section{Introduction}

In May 2016, a community outbreak of invasive group A streptococcus (iGAS) was declared by the Medical Officer of Health of the Middlesex-London Health Unit, a ruralurban Ontario community with fewer than 500,000 people. Despite applying current Canadian guidelines for institutional outbreaks-which were adapted for this community outbreakthe outbreak is ongoing. The purpose of this report is to describe this community outbreak: the epidemiology of reported cases, the public health response and the challenges that arose when attempting to apply the institutional-based guidelines.

\section{Background}

Group A streptococci (GAS) are human bacterial pathogens that colonize the throat or skin and may be present in asymptomatic carriers. This gram-positive bacteria can cause a broad spectrum of disease that can be noninvasive or invasive. Noninvasive disease includes pharyngitis (e.g., strep throat), impetigo, scarlet fever and cellulitis $(1,2)$. Invasive group A streptococcal infections (iGAS) occur when the pathogens infect normally sterile sites, such as the blood, cerebrospinal fluid, joints, pleural or pericardial fluid $(1,3)$. Text box 1 summarizes the Ontario Ministry of Health and Long-Term Care provincial case definition for a laboratory-confirmed case of iGAS (3). Although uncommon 


\section{Text box 1: Definition of a laboratory-confirmed case of invasive group A streptococcia}

Isolation of group A streptococcus (Streptococcus pyogenes) or DNA detection by nucleic acid amplification test (NAAT) from a normally sterile site (e.g., blood, cerebrospinal fluid, joint fluid, pleural fluid, pericardial fluid) with or without evidence of clinical severity, OR

Isolation of group A streptococcus from a nonsterile site (e.g., skin) with evidence of severity. Clinical severity is defined as one of the following:

- Streptococcal toxic shock syndrome (STSS), which is characterized by hypotension (systolic blood pressure $<90 \mathrm{~mm}$ $\mathrm{Hg}$ in adults or $<5$ th percentile for age for children) and at least two (2) of the following signs:

- renal impairment (creatinine $>177 \mu \mathrm{mol} / \mathrm{L}$ for adults)

- coagulopathy (platelet count $\leq 100,000 \mathrm{~mm}^{3}$ or disseminated intravascular coagulation):

- liver function abnormality (AST (SGOT), ALT (SGPT) or total

bilirubin $\geq 2 x$ upper limit of normal for age);

- adult respiratory distress syndrome (ARDS);

- generalized erythematous macular rash that may desquamate; OR

- Soft tissue necrosis, including necrotizing fasciitis or myositis or gangrene; OR

- Meningitis; OR

- Death; OR

- A combination of any of these conditions.

Abbreviations: ALT, alanine aminotransferase; AST, aspartate aminotransferase; DNA, deoxyribonucleic acid; SGOT, serum glutamic-oxaloacetic transaminase; SGPT, serum glutamic pyruvic transaminase

Pro

(http://www.health.gov.on.ca/en/pro/programs/publichealth/oph_standards/docs/gas_cd.pdf)

compared to noninvasive GAS infection, iGAS is associated with significant morbidity and mortality, as the bacteria can cause severe sequelae such as pneumonia, meningitis, necrotizing fasciitis, sepsis and streptococcal toxic shock syndrome $(4,5)$. For GAS isolates, the M protein (encoded by the emm gene) is a significant virulence and epidemiologic factor that impacts the pathogen's ability to evade the human immune response to infection (6).

Historically, outbreaks of iGAS have occurred in long-term care facilities (7-10), hospitals (8) and child care centres (11-14). The Public Health Agency of Canada's 2006 Guidelines for the Prevention and Control of Invasive Group A Streptococcal Disease focus on managing disease in these facilities (15). Subsequent provincial protocols $(3,16)$ and recommendations (17) have also focused on these settings.

However, recent outbreaks of both noninvasive and invasive GAS disease have been reported in community settings, often in marginalized populations. For example, there have been reports of GAS outbreaks in a homeless shelter in Toronto, Ontario (18), a homeless population in Alaska, United States (US) (19), persons who use drugs (PWUD) in England and Wales (20), and a population with a high prevalence of PWUD, alcohol abuse and homelessness in Thunder Bay, Ontario (21).

The Canadian guidelines for institutional iGAS outbreaks include a retrospective chart review, identification of close contacts, alerting contacts to signs and symptoms, strict enforcement of standard infection control practices and a 10day chemoprophylaxis of close contacts of a confirmed severe case (15). However, these guidelines are difficult to implement in marginalized populations.
In 2004, the United Kingdom's Health Protection Agency released interim guidelines for the management of community-acquired cases of iGAS (22). While the guidelines acknowledge that cases occurring in homeless shelters can present a challenge for public health action, the recommendations do not include additional measures for marginalized populations. For example, there are no measures addressing barriers to accessing primary health care, to reaching those affected (e.g., those with no personal phone number and with no fixed address), or to getting full disclosure regarding contacts or challenges in completing a 10-day course of treatment (16).

\section{Methods}

\section{Outbreak detection and investigation}

Between January 2015 and March 2016, the average monthly incidence of iGAS in the Middlesex-London Health Unit was 1.73 cases per month overall, and 0.47 cases per month among PWUD and/or under-housed individuals. In a 25-day period spanning April and May 2016, five cases of iGAS were reported to the Middlesex-London Health Unit, all who were PWUD; this amounted to more than two standard deviations (SD) of the monthly mean for overall cases $(2 \mathrm{SD}+$ mean $=3.80)$ and for PWUD and/or under-housed cases (2 SD + mean $=1.50)$. Due to the potential for iGAS to have severe clinical outcomes (including death), the Middlesex-London Health Unit declared a community outbreak of iGAS in its jurisdiction on May 12, 2016.

The outbreak case definition included all cases of laboratory-confirmed iGAS in Middlesex-London with an accurate episode date on or after April 1, 2016. The accurate episode date is the earliest recorded date of symptom onset, date of lab specimen collection or date reported to public health. This article includes cases reported to the Middlesex-London Health Unit up to and including February 28, 2018.

In Ontario, molecular typing is not routinely conducted on iGAS specimens. As part of the outbreak investigation, laboratory specimens were sent to the Public Health Ontario Laboratories for pulsed-field gel electrophoresis (PFGE) typing and to the National Microbiology Laboratory for emm typing.

\section{Contact tracing}

Contact tracing focused on individuals in close contact with iGAS cases, defined according to the Public Health Agency of Canada's Guidelines for the Prevention and Control of Invasive Group A Streptococcal Disease (15) (summarized in Text box 2). Middlesex-London Health Unit staff attempted to contact all the close contacts identified to alert them to the signs and symptoms of iGAS, to advise them to seek medical attention should they develop any clinical signs of iGAS and to determine whether chemoprophylaxis might be warranted.

\section{Risk factor assessment}

Anyone who reported illicit drug use (e.g., opioids, cocaine, methamphetamines) during the six months preceding the diagnosis was considered a "PWUD"; exclusive users of marijuana were excluded. "Under-housed" was defined as an individual with no fixed address, living in a homeless shelter or group home or couch-surfing (i.e. temporarily staying with friends). To better understand whether drug use practices may be contributing to iGAS transmission, an enhanced drug 
Textbox 2: Definition of close contacts of iGAS cases (15)

- Household contacts of a case who have spent at least 4 hours/ day on average in the previous 7 days or 20 hours/week with the case;

- Non-household contacts who shared the same bed with the case or had sexual relations with the case;

- Contacts who had direct mucous membrane contact with the oral or nasal secretions of a case (e.g., mouth-to-mouth resuscitation, open mouth kissing) or had unprotected direct contact with an open skin lesion of the case;

- Injection drug users who shared needles with the case.

Abbreviation: iGAS, invasive group A streptococcal

questionnaire was developed, with input from community partners, for PWUD iGAS cases. The questionnaire was piloted in April 2017 and administered between May 1, 2017, and January 31, 2018 (available upon request).

\section{Environmental health inspections}

To assess the living conditions at premises linked to cases, Middlesex-London Health Unit public health inspectors performed site visits to unlicensed rooming houses (a house or building where multiple tenants share kitchen and/or washroom facilities and each tenant pays individual rent) and a licensed lodging home. Site visits to local homeless shelters were also conducted to determine client interaction, facility sanitation and cleaning, the use of laundry and shower facilities, and the availability of health care services for clients.

\section{Data analysis}

A case line-list was extracted from the integrated Public Health Information System (iPHIS) and imported into Microsoft Excel (version 2010; Microsoft Inc, Redmond, Washington, US). The line-list included information on age, sex, address, episode date and laboratory test results. Additional information contained in the internal Middlesex-London Health Unit Infectious Disease Control database (e.g., drug use, housing, existing conditions, symptoms, clinical severity) were added to the spreadsheet. Analyses were conducted in Microsoft Excel and STATA/SE (version 14.0; StataCorp, College Station, Texas, US). Rate ratios (RR) and Pearson chi-squared test (or Fisher exact test) were used to assess the difference in proportions between groups.

\section{Results}

A total of 156 confirmed iGAS cases with an accurate onset date between April 1, 2016, and February 28, 2018, were reported to the Middlesex-London Health Unit. This corresponded to 147 people because nine individuals had two separate episodes during the 23-month period. One individual with a recent case of iGAS was identified as a potential close contact of another case (i.e. the contact may have been the index case); otherwise, no secondary cases of iGAS were reported among close contacts. Figure 1 shows an epidemic curve of cases by month of onset from January 1, 2015, to February 28, 2018.

The cases were between three and 98 years of age (median: 47); there were more male $(91 / 147 ; 61 \%)$ than female cases (Table 1$)$.

Cases of iGAS were reported among two different groups of people. In the first group, approximately half the cases (48\%; 71/147) were marginalized populations defined as PWUD and/or under-housed. Of those, 29 (41\%) were exclusively PWUD, six (8\%) were exclusively under-housed and 36 (51\%) were both. Among the PWUD and/or under-housed cases $(n=71)$, most were in the $30-39$ year age group $(20 / 71 ; 28 \%)$. In addition, $52(73 \%)$ were co-infected with the hepatitis $\mathrm{C}$ virus (HCV) and $15(21 \%)$ with the human immunodeficiency virus (HIV). Nearly all the cases $(14 / 15 ; 93 \%)$ with HIV were co-infected with HCV. For those who were not aware of their HCV or HIV status, testing performed at the time of treatment for iGAS resulted in newly diagnosed cases of HCV $(n=8)$ and HIV $(n=3)$, with care for these conditions initiated at the hospital.

The second group consisted of people who were neither PWUD nor under-housed $(n=76)$. Approximately $22 \%$ were aged $50-59$ years and represented the largest age group (17/76). Slightly

Figure 1: Cases of invasive group A streptococcal infection in Middlesex-London, Ontario, by month of onset (January 1, 2015-February 28, 2018)

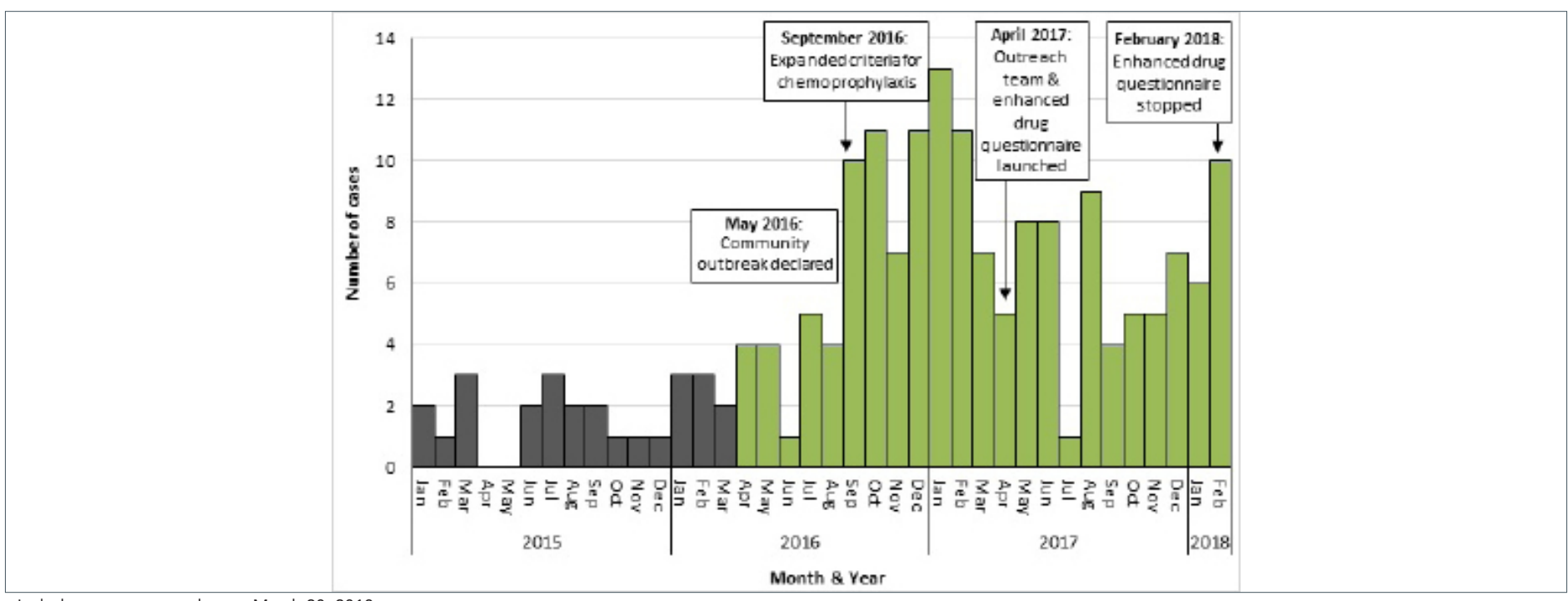

Includes cases reported up to March 20, 2018

Legend: Green bars denote cases included in the outbreak investigation (from April 1, 2016, onwards). Grey bars denote cases with an onset date prior to the outbreak 
Table 3: Distribution of emm types of cases of invasive group A streptococcal infection in Middlesex-London, Ontario, by drug use and/or under-housed status, April 1, 2016-February 28, 2018

\begin{tabular}{|l|r|r|}
\hline \multicolumn{1}{|c|}{ emm type } & $\begin{array}{c}\text { PWUD and/or } \\
\text { under-housed } \\
\mathrm{n}(\%)\end{array}$ & $\begin{array}{c}\text { Neither PWUD nor } \\
\text { under-housed } \\
\mathrm{n}(\%)\end{array}$ \\
\hline emm1 & $1(2)$ & $14(29)$ \\
\hline emm4 & 0 & $6(12)$ \\
\hline emm5 & 0 & $1(2)$ \\
\hline emm6 & 0 & $1(2)$ \\
\hline emm9 & $11(18)$ & $5(10)$ \\
\hline emm12 & 0 & $2(4)$ \\
\hline emm28 & 0 & $2(4)$ \\
\hline emm73 & $2(3)$ & $2(4)$ \\
\hline emm74 & $18(29)$ & $6(12)$ \\
\hline emm77 & $2(3)$ & 0 \\
\hline emm81 & $27(44)$ & $7(14)$ \\
\hline emm87 & $1(2)$ & 0 \\
\hline emm89 & 0 & $3(6)$ \\
\hline Total & 62 & 49 \\
\hline
\end{tabular}

Abbreviations: N/A, not applicable; $n$, number; PWUD, persons who use drugs

a Includes results received by the Middlesex-London Health Unit by March 20, 2018

$(111 / 156 ; 71 \%)$

physician newsletter, notices to community partners and posters in needle exchange facilities, shelters and clinics that serve PWUD and/or under-housed clients.

The Middlesex-London Health Unit also continued to promote harm reduction practices to prevent transmission (e.g., not sharing needles, using alcohol swabs at the injection site, not licking skin or needle tip before injecting, heating drugs before injecting) and encouraged clinical referrals to treat possible strep throat, cellulitis or wound infections. Meetings with stakeholders were also organized to provide updates about the outbreak and discuss strategies to detect cases early on and initiate referrals for wound care (e.g., training on how to spot wounds that require medical attention).

\section{Environmental health inspections}

Site visits to unlicensed rooming houses uncovered unsanitary living conditions, large quantities of needle waste, infestations of bedbugs and cockroaches, and structural defects (e.g., broken fixtures, exposed electrical wiring, holes in the walls). As a result, a number of directives were issued by the public health inspectors that included pest control, environmental cleaning and safe collection and disposal of needle waste.

Health unit investigators noted that a large number of shelter clients had visible open wounds on their faces and forearms. At the shelter associated with the highest number of cases, shared spaces were regularly cleaned and clients had access to shower and laundry facilities; however, emergency beds were found to be in close proximity to each other. A lodging home that had two cases of iGAS and one case of GAS was found to have satisfactory sanitation conditions.
Adapting strategies for contact tracing and chemoprophylaxis In September 2016, the Middlesex-London Health Unit expanded its criteria for chemoprophylaxis to include contacts who shared drugs or injection equipment with a known iGAS case, regardless of the case's clinical severity. The possibility of offering chemoprophylaxis broadly to local homeless shelter clients was considered in order to reduce asymptomatic carriage rates. However, more than half of the under-housed cases (22/42) did not report spending time in homeless shelters, and cases associated with shelters were scattered in time a nd across different shelters and involved at least four different emm types. Consequently, the decision was made to not move forward with this intervention, considering the limited reach, the resources that would have been required to implement it and the potential health risks. In April 2017, the Middlesex-London Health Unit revised its antibiotic chemoprophylaxis recommendations to offer single-dose azithromycin to individuals who had had close contact with PWUD and/or under-housed cases as the recommended 10-day regimen was potentially difficult for this vulnerable population to complete.

During the outbreak, the Middlesex-London Health Unit Outreach Team was launched to help identify and provide street-level support to people who were under-housed or used drugs. The goal was to help reduce the transmission of infection among this population. The Outreach Team located, engaged with and educated clients, and linked them to care, treatment and basic needs programs (e.g., housing, Infectious Disease Care Program). Initially, the Team also administered the enhanced drug questionnaire to PWUD cases, but stopped doing so because of the significant resources required to administer the survey and its similarity to another project. Moreover, findings from the initial questionnaires failed to add new insights about drug injection practices.

\section{Discussion}

This 23-month outbreak of iGAS in a rural/urban community in Ontario has affected two distinct populations and is yet to be controlled. Although the number of reported iGAS cases reached a peak in January 2017, the monthly case counts continue to be higher than the previous 5-year monthly average. Reason(s) for the increased incidence of iGAS in the Middlesex-London Health Unit remains unclear. Unlike outbreaks reported in Toronto, Ontario (18), Montréal, Quebec (23) and Anchorage, Alaska (19), few epidemiologic links have connected the cases reported in the Middlesex-London Health Unit (e.g., matching isolate strains, presence in the same location). For example, 13 different emm types have been reported to date and cases have been distributed across different locations within the health unit. While nearly half of the cases have involved PWUD and under-housed individuals, the incidence rate has also increased among seemingly unrelated individuals living in stable housing and with no history of drug use. Findings from studies in the United States (24) and United Kingdom (25) have also found that cases among people who inject drugs tend to have less clinically severe infections than among people who do not inject drugs.

There were a number of challenges in applying the existing Guidelines for the Prevention and Control of Invasive Group A Streptococcal Disease. For example, although criteria have been developed to trigger action when iGAS occurs in a long-term care facility, hospital or child care centre (e.g., one severe case of iGAS in a child attending a child care centre) (15), criteria have not been developed for cases or clusters within a community. 
A number of cases who were PWUD and/or under-housed were difficult to locate or could not be tracked down for investigation and follow-up (e.g., no phone, no fixed address). When cases were contacted, interviews were often challenging: some individuals were reluctant or refused to speak to public health and/or hospital staff, especially about their drug use and practic es. Contact tracing for PWUD cases was often difficult as many individuals were reluctant or unwilling to disclose the name of their needle-sharing partners. Some cases who were either too ill or not sufficiently cogent to respond required multiple interview attempts.

In addition, possible adaptations of the guidelines for the community - such as routine testing to determine GAS and iGAS colonization rates or more widespread chemoprophylaxis in an under-housed population-have yet to be evaluated to determine effectiveness (26). For example, current guidelines identify first-generation cephalosporins (e.g., cephalexin) as the preferred antibiotic for iGAS chemoprophylaxis (15). This regimen requires a 10-day treatment, which may be difficult for PWUD and/or under-housed individuals to complete. To control an iGAS outbreak at six homeless service sites in Anchorage, Alaska, public health authorities offered a single-dose regimen (azithromycin) to clients, staff and volunteers (19). In Canada, azithromycin is not recommended as a first- or second-line treatment because evidence shows that it may select for macrolide resistance more strongly than either erythromycin or clarithromycin $(15,27)$.

For the current outbreak, it is unclear whether chemoprophylaxis has been effective in preventing secondary cases. Expanding the criteria for the chemoprophylaxis of contacts and offering a single-dose regimen (azithromycin) did not appear to have resulted in any direct reduction in the incidence of iGAS.

Furthermore, the emergence of macrolide-resistant $S$. pyogenes emm9 strains may indicate greater selective pressure after these adaptations to the guidelines were implemented. To prevent further development of antimicrobial resistance, the Middlesex-London Health Unit will explore alternatives to azithromycin for chemoprophylaxis of contacts who may have trouble adhering to a 10-day regimen. Alternatives could include injectable penicillin or directly observed therapy with outreach workers.

The data included in this report are subject to a number of limitations. Because disease investigations were largely based on case interviews, the information is subject to recall and self-reporting bias. As drug use, precarious housing and other potentially high-risk behaviours (e.g., sexual activities) were likely underreported, some cases may have been misclassified during the investigation. As well, emm and PFGE typing data for cases were incomplete; due to communication and procedural issues, a number of specimens were not forwarded from the hospital laboratory to the provincial laboratory for molecular testing.

Other clusters of iGAS are currently being investigated across Ontario and each have somewhat different epidemiologic characteristics (i.e. different emm types, different affected populations). Information about the current outbreak in Middlesex-London as well as the clusters occurring elsewhere may help public health officials understand the reason(s) for the increase in the incidence of iGAS, and determine potential public health interventions that may be effective in preventing and mitigating future outbreaks. Trends in antimicrobial resistance also need to be monitored.

\section{Conclusion}

There are unique challenges to controlling iGAS outbreaks in the community, especially when they involve marginalized populations. There is a need for specific guidelines for managing outbreaks of iGAS in the community-including among marginalized populations. Until then, public health efforts will continue to focus on contact tracing to identify those at a higher risk of acquiring iGAS in order to be able to prevent new cases or to treat infections at an early stage, and thus prevent further transmission.

\section{Authors' statement}

$C D$ conceived the first draft of this manuscript. $C D, K K$ and MP analyzed the data. CD, CB, VN and GH were involved in the outbreak investigation and public health management. All authors contributed to the development and revision of this manuscript.

\section{Conflict of interest}

None.

\section{Acknowledgements}

Our thanks to the Public Health Agency of Canada for mobilizing a field epidemiologist to assist in the outbreak investigation; Mary Lou Albanese and the Middlesex-London Health Unit staff who assisted with the investigation, including case follow-up and contact tracing, the Public Health Ontario Laboratories for conducting PFGE typing and the National Microbiology Laboratory for conducting emm typing. We also thank our community partners who provided guidance and assisted the Middlesex-London Health Unit in raising awareness about iGAS, as well as Drs Michael John, Michael Finkelstein, Emily Mosites, Louisa Castrodale and Fiona Kouyoumdjian for their advice and guidance.

\section{References}

1. Cunningham MW. Pathogenesis of group A streptococcal infections. Clin Microbiol Rev 2000 Jul;13(3):470-511. http:// dx.doi.org/10.1128/CMR.13.3.470-511.2000. PubMed (https:// www.ncbi.nlm.nih.gov/pmc/articles/PMC88944/)

2. World Health Organization. The current evidence for the burden of group A streptococcal diseases. Geneva: WHO: 2005. http://www.who.int/maternal_child_adolescent/ documents/fch_cah_05_07/en/

3. Ontario Ministry of Health and Long-Term Care. Infectious disease protocol. Appendix B: Provincial case definitions for reportable diseases. Disease: group A streptococcal disease, invasive (iGAS). Toronto: Ontario Ministry of Health and Long-Term Care. http://www.health.gov.on.ca/en/pro/ programs/publichealth/oph_standards/docs/gas_cd.pdf

4. Meehan M, Murchan S, Bergin S, O'Flanagan D, Cunney R. Increased incidence of invasive group $A$ streptococcal disease in Ireland, 2012 to 2013. Euro Surveill 2013 Aug;18(33):20556. http://dx.doi.org/10.2807/1560-7917.ES2013.18.33.20556. PubMed (https://www.ncbi.nlm.nih.gov/pubmed/23968875) 
5. Rudolph K, Bruce MG, Bruden D, Zulz T, Reasonover A, Hurlburt D, Hennessy T. Epidemiology of invasive group A streptococcal disease in Alaska, 2001 to 2013. J Clin Microbiol 2016 Jan;54(1):134-41. http://dx.doi.org/10.1128/ JCM.02122-15. PubMed (https://www.ncbi.nlm.nih.gov/ pubmed/26560536)

6. Hraoui M, Boutiba-Ben Boubaker I, Doloy A, Samir E, Ben Redjeb S, Bouvet A. Epidemiological markers of Streptococcus pyogenes strains in Tunisia. Clin Microbiol Infect 2011 Jan;17(1):63-8. http://dx.doi.org/10.1111/j.14690691.2010.03174.x. PubMed (https://www.ncbi.nlm.nih.gov/ pubmed/20132259)

7. Harkness GA, Bentley DW, Mottley M, Lee J. Streptococcus pyogenes outbreak in a long-term care facility. Am J Infect Control 1992 Jun;20(3):142-8. http://dx.doi.org/10.1016/ S0196-6553(05)80181-6. PubMed (https://www.ncbi.nlm.nih. gov/pubmed/1636935)

8. Schwartz B, Elliott JA, Butler JC, Simon PA, Jameson BL, Welch GE, Facklam RR. Clusters of invasive group A streptococcal infections in family, hospital, and nursing home settings. Clin Infect Dis 1992 Aug;15(2):277-84. http://dx.doi.org/10.1093/ clinids/15.2.277. PubMed (https://www.ncbi.nlm.nih.gov/ pubmed/1520763)

9. Centers for Disease Control (CDC). Nursing home outbreaks of invasive group A streptococcal infections--Illinois, Kansas, North Carolina, and Texas. MMWR Morb Mortal Wkly Rep 1990 Aug;39(34):577-9. PubMed (https://www.ncbi.nlm.nih. gov/pubmed/2117240)

10. Barnham M, Hunter S, Hanratty B, Kirby P, Tanna A, Efstratiou A. Invasive M-type 3 Streptococcus pyogenes affecting a family and a residential home. Commun Dis Public Health 2001 Mar;4(1):64-7. PubMed (https://www.ncbi.nlm.nih.gov/ pubmed/11467024)

11. Aguero J, Ortega-Mendi M, Eliecer Cano M, Gonzalez de Aledo A, Calvo J, Viloria L, Mellado P, Pelayo T, Fernandez-Rodriguez A, Martinez-Martinez L. Outbreak of invasive group A streptococcal disease among children attending a day-care center. Pediatr Infect Dis J 2008 Jul;27(7):602-4. http://dx.doi.org/10.1097/ INF.0b013e31816a0e0a. PubMed (https://www.ncbi.nlm.nih. gov/pubmed/18520444)

12. Centers for Disease Control and Prevention (CDC). Outbreak of invasive group A Streptococcus associated with varicella in a childcare center -- Boston, Massachusetts, 1997. MMWR Morb Mortal Wkly Rep 1997 Oct;46(40):944-8. PubMed (https:// www.ncbi.nlm.nih.gov/pubmed/9338455)

13. Falck G, Kjellander J. Outbreak of group A streptococcal infection in a day-care center. Pediatr Infect Dis J 1992 Nov;11(11):914-9. http://dx.doi.org/10.1097/00006454199211110-00002. PubMed (https://www.ncbi.nlm.nih.gov/ pubmed/1454431)

14. Engelgau MM, Woernle CH, Schwartz B, Vance NJ, Horan JM. Invasive group A streptococcus carriage in a child care centre after a fatal case. Arch Dis Child 1994 Oct;71(4):318-22. http:// dx.doi.org/10.1136/adc.71.4.318. PubMed (https://www.ncbi. nlm.nih.gov/pubmed/7979524)

15. Public Health Agency of Canada. Guidelines for the prevention and control of invasive group A streptococcal disease. Can Commun Dis Rep 2006;32(Suppl 2):1-26. https://www.canada. $\mathrm{ca} / \mathrm{en} /$ public-health/services/reports-publications/canadacommunicable-disease-report-ccdr/monthly-issue/2006-32/ canada-communicable-disease-report.html

16. Ontario Ministry of Health and Long-Term Care. Infectious disease protocol. Appendix A: Disease-specific chapters. Chapter: Group A streptococcal disease, invasive. Toronto (ON): Queen's Printer for Ontario. http://www.health.gov. on.ca/en/pro/programs/publichealth/oph_standards/docs/ gas_chapter.pdf
17. Public Health Ontario. Recommendations on public health management of invasive group A streptococcal (iGAS) disease. Provincial Infectious Diseases Advisory Committee. Toronto (ON): Queen's Printer for Ontario. Toronto (ON): 2014 Sep. https://www.publichealthontario.ca/en/eRepository/iGAS_ Recommendations_on_Public_Health_Management.pdf

18. Finkelstein M. ProMED mail: Streptococcus, group A - Canada (02): (ON) fatal, homeless shelter emm74, clonal. Brookline (MA): International Society for Infectious Diseases; 2017 Apr 5. www.promedmail.org/post/20170406.4952247

19. Mosites E, Frick A, Gounder P, Castrodale L, Li Y, Rudolph K, Hurlburt D, Lecy KD, Zulz T, Adebanjo T, Onukwube J, Beall B, Van Beneden CA, Hennessy T, McLaughlin J, Bruce MG. Outbreak of invasive infections from subtype emm26.3 group A streptococcus among homeless adults-Anchorage, Alaska, 2016-2017. Clin Infect Dis 2018 Mar;66(7):1068-74. http:// dx.doi.org/10.1093/cid/cix921. PubMed (https://www.ncbi. $\mathrm{nlm.nih.gov/pubmed/29069346)}$

20. Bundle N, Bubba L, Coelho J, Kwiatkowska R, Cloke R, King S, Rajan-lyer J, Courtney-Pillinger M, Beck CR, Hope V, Lamagni T, Brown CS, Jermacane D, Glass R, Desai M, Gobin M, Balasegaram S, Anderson C. Ongoing outbreak of invasive and non-invasive disease due to group A Streptococcus (GAS) type emm66 among homeless and people who inject drugs in England and Wales, January to December 2016. Euro Surveill 2017 Jan;22(3):30446. http://dx.doi.org/10.2807/1560-7917. ES.2017.22.3.30446. PubMed (https://www.ncbi.nlm.nih.gov/ pmc/articles/PMC5322289/)

21. Athey TB, Teatero S, Sieswerda LE, Gubbay JB, Marchand-Austin A, Li A, Wasserscheid J, Dewar K, McGeer A, Williams D, Fittipaldi N. High incidence of invasive group A Streptococcus disease caused by strains of uncommon emm types in Thunder Bay, Ontario, Canada. J Clin Microbiol 2016 Jan;54(1):83-92. http://dx.doi.org/10.1128/JCM.02201-15. PubMed (https://www.ncbi.nlm.nih.gov/pubmed/26491184)

22. Health Protection Agency, Group A Streptococcus Working Group. Interim UK guidelines for management of close community contacts of invasive group A streptococcal disease. Commun Dis Public Health 2004 Dec;7(4):354-61. PubMed (https://www.ncbi.nlm.nih.gov/pubmed/15786581)

23. Savard N. ProMED mail: Streptococcus, group A - Canada (03): (QC) fatal, homeless, emm74, RFI. Brookline (MA): International Society for Infectious Disease; 2017 Aug 15. http://outbreakwatch.blogspot.ca/2017/08/proedrstreptococcus-group-canada-03-qc.html

24. Navarro VJ, Axelrod PI, Pinover W, Hockfield HS, Kostman JR. A comparison of Streptococcus pyogenes (group A streptococcal) bacteremia at an urban and a suburban hospital. The importance of intravenous drug use. Arch Intern Med 1993 Dec;153(23):2679-84. http://dx.doi.org/10.1001/ archinte.1993.00410230097011. PubMed (https://www.ncbi. nlm.nih.gov/pubmed/8250663)

25. Lamagni TL, Neal S, Keshishian C, Alhaddad N, George $R$, Duckworth G, Vuopio-Varkila J, Efstratiou A. Severe Streptococcus pyogenes infections, United Kingdom, 20032004. Emerg Infect Dis 2008 Feb;14(2):202-9. http://dx.doi. org/10.3201/eid1402.070888. PubMed (https://www.ncbi.nlm. nih.gov/pmc/articles/PMC2600190/)

26. Alaska Department of Health and Social Services. Alaska Public Health Advisory: invasive group A streptococcal infections caused by a novel emm type. Anchorage (AK): State of Alaska; 2016 Nov 29. http://dhss.alaska.gov/dph/Epi/Documents/ phan/GAS\%20PHAN_nov_29_2016.pdf

27. Vanderkooi OG, Low DE, Green K, Powis JE, McGeer A; Toronto Invasive Bacterial Disease Network. Predicting antimicrobial resistance in invasive pneumococcal infections. Clin Infect Dis 2005 May;40(9):1288-97. http://dx.doi. org/10.1086/429242. PubMed (https://www.ncbi.nlm.nih.gov/ pubmed/15825031) 